\title{
Analysis and selection of Harmonics sensitive to Demagnetization Faults intended for Condition Monitoring of Double Rotor Axial Flux Permanent Magnet Synchronous Machines \\ ISSN 1751-8644 doi: 0000000000 www.ietdl.org
}

\author{
Jan De Bisschop ${ }^{1,2}$ Ahmed Abou-Elyazied Abdallh ${ }^{2}$ Peter Sergeant ${ }^{1,2}$ Luc Dupré $^{1,2}$ \\ ${ }^{1}$ Department of Electrical Energy, Metals, Mechanical Constructions \& Systems, Ghent University, Belgium \\ ${ }^{2}$ Flanders Make, the strategic research centre for the manufacturing industry \\ E-mail: jan.debisschop@ugent.be
}

\begin{abstract}
Yokeless and Segmented Armature (YASA) Axial Flux Permanent Magnet Synchronous Machines may have asymmetrical demagnetization defects in their two rotors. Condition monitoring therefore becomes more complicated than in conventional single rotor machines. In order to develop a real-time condition monitoring algorithm for demagnetization faults in YASA machines - which requires solving an inverse problem - a fast forward model is necessary. A frequency-based analytical model is used to calculate the three phase terminal voltages based on the three phase current waveforms and the demagnetization defects, including asymmetrical defects in the two rotors. In order to reduce the computational time of the forward model, the paper focuses on the sensitivity of different voltage harmonics on several demagnetization faults. To this end, the Cramér-Rao lower bound technique is used to analyse the harmonics sensitivity to the demagnetization faults. The goal of the sensitivity analysis is to select an appropriate set of harmonics that gives maximal information about the defects. It becomes much faster and useful for real-time condition monitoring. The results of the analysis show that the subharmonics around and including the fundamental are the most sensitive harmonics to the demagnetization defects. The analytical model is validated with experimental data and finite element simulations.
\end{abstract}

\section{Introduction}

Axial flux permanent magnet synchronous machines (AFPMSMs) of the yokeless and segmented armature type (YASA) have been extensively used in different applications thanks to their excellent performances [1][2][3]. Particularly, AFPMSMs are well dedicated for applications that acquire high power density, such as sustainable energy applications. In practice, these types of machines may suffer from different faults, such as rotor eccentricity and permanent magnet (PM) demagnetization, which decrease their reliability [4][5]. In order to prevent the defect from progressing and creating more damage in the machine, online condition monitoring systems are highly needed to detect the faults in early stages.

The demagnetization defects may occur due to an excessive temperature rise in the rotor of the machine, or due to high load currents or short circuit currents in the stator. When magnets are exposed to high temperatures, their magnetization may be lost partially or completely [6][7]. This problem can be mitigated by using magnets of a higher temperature class, but these magnets are more expensive and have a lower remanence. A better solution is to detect the demagnetization via a real-time monitoring system.

Demagnetization faults can be detected on-line by several sensor and/or sensorless techniques. In [8] and [9], an overview of these techniques is given. Four types of detection techniques are identified. The first type consists of current-based indexes. Here the current waveforms are analysed in either the time or the frequency domain $[10][11][12][13]$. Some techniques require machine standstill while others require steady-state operation. If the machine has parallel branches in the stator phase windings, the current components for the separate branches can be examined as well. The second type of indexes are the voltage-based indexes, where either the instantaneous back-EMF [14] or the fundamental component of the zero-sequence voltage component is examined [15]. Although the proposed current and voltage indexes are fast to calculate, they have some disadvantages too. They rely on a priori knowledge of the current or voltage waveforms. This knowledge is usually based on time-consuming numerical models. This limits the flexibility toward a variation of the technique in parameters or topology. Furthermore, standstill and steady-state operations are requirements which cannot be met in every situation. In case of the zero-sequence voltage components techniques, a fourth leg in the converter and an additional current sensor are required, which increases the cost. The third type are the torque-based indexes. Demagnetization increases the torque ripple, especially the amplitude of the sideband components (ASBCs) of the torque. These side bands can be measured with a torque meter and used for detection [16]. An alternative is to perform a Time Delay Embedding (TDE) analysis of the torque time signals and to analyse the resulting 2-D representation [17]. Another possibility is to either estimate [18] or measure [19] the torque constant of the machine. Finally, the fourth type are the magnetic flux-based indexes. Either the magnetic flux is measured with a Gauss meter or a flux probe [20][21] or it is analysed using an analytical model [22]. Both torque measurements and magnetic flux measurements introduce an extra cost of the sensors.

A YASA AFPMSM has two rotors, which complicates the modelling and the detection of demagnetization problems in comparison to machines with one rotor. Indeed, in this dual-rotor machine, asymmetric defects can occur, which means that demagnetization can be different for every rotor. This makes condition monitoring different from other work in literature for machines with one rotor.

For this machine, an inverse problem (IP) based methodology was presented to detect demagnetization faults [22]. This IP interprets the measured voltage waveforms into a frequency-based analytical model that takes the PM demagnetization into consideration. This analytical model solves Maxwell's equations for a predefined number of time and space harmonics. Modelling few harmonic orders leads to inaccurate representations of the machine electromagnetic 


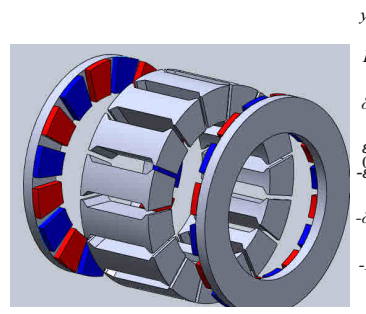

(a)

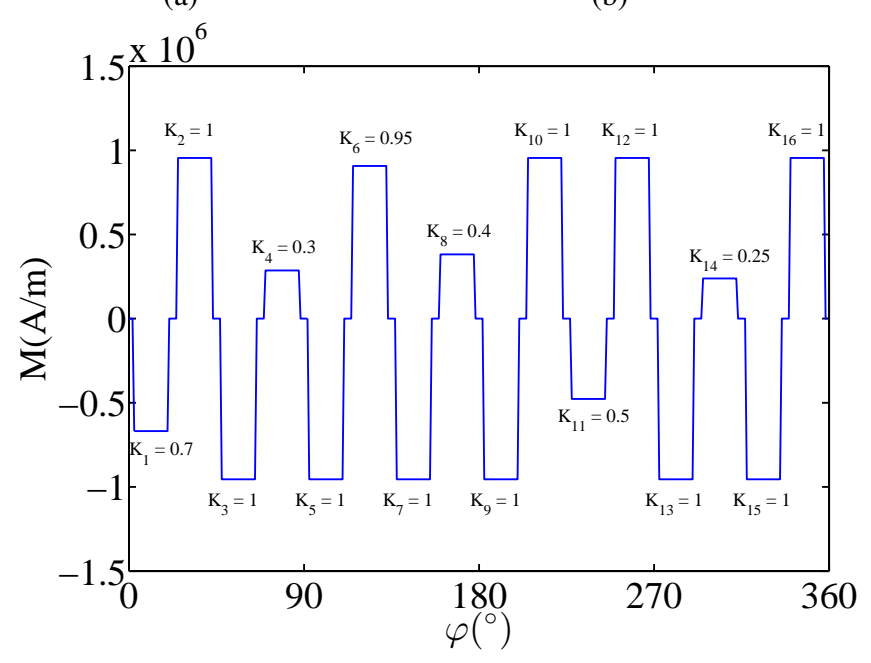

(c)

Fig. 1: (a) The exploded view of the studied AFPMSM. (b) Analytical model for solving of no-load magnetic field of AFPMSM showing the different regions. In the model, the stator is represented as a solid isotropic cylinder with an axial length $\varepsilon$, which is chosen in such a way that the tangential reluctance of the model equals the reluctance of the real stator with slots. (c) Shows an example of a magnetization pattern with corresponding magnetization factors for each magnet of the right rotor with $p=8$.

behaviour, and consequently, inaccurate identification results. On the contrary, modelling many harmonic orders dramatically increases the computational time. Hence, there is a need to identify which time and space harmonic orders are the most useful to solve the inverse problem fast and accurately.

In this paper, we aim at analysing and determining the optimal set of time harmonics that needs to be simulated for a better detection of PM demagnetization faults in the AFPMSM. First, the frequencybased analytical model is outlined in section 2 . Second, section 3 identifies the set of time harmonics that is most sensitive to demagnetization faults. The inverse problem is formulated in section 3.1. Section 3.2 presents a theoretical framework about the expected time harmonics that can appear in healthy and faulty machines and section 3.3 applies this in a practical case study. The Cramér-Rao lower bound (CRLB) technique, which is utilized recently in electromagnetic applications [23], is implemented to analyse the sensitivity of the time harmonics to a defect in section 4.1 of which the results are given in sections 4.2, 4.3 and 4.4. Finally, the conclusion is drawn in section 5 .

\section{A frequency-based analytical model}

\subsection{Analytical five-region model of the AFPMSM}

This model is developed to investigate the demagnetization defects in YASA type AFPMSMs. The topology of these machines is shown in Fig. 1(a). Using the same analytical subdomain modelling approach as the three-region model of [22], a five-region model of the machine is developed. Compared to the three-region model of [22], the five-region model describes in a more accurate way asymmetrical demagnetization faults in both rotors. Asymmetrical demagnetization occurs when the magnets of both rotors are not demagnetized equally. In the healthy state and with symmetrical defects, the flux density vector in the stator is purely in axial direction. In contrast to this, asymmetrical defects will require closing paths tangential through the stator. In the three-region model, the stator is not modelled, and the tangential closing path in the stator cannot be modelled. The five-region model however includes a simplified stator as region III. This allows for flux paths to close through the stator, resulting in more accurate flux density fields in the air gaps, especially in case of asymmetrical defects. Note that the analytical subdomain model can also be combined with a Magnetic Equivalent Circuit such as in [24], making it more accurate but also slower.

Fig. 1(b) shows the five-region model for simulating the no-load magnetic field of the AFPMSM. A calculation plane is created from a cylindrical cross section with a constant radius. For this plane, a Cartesian coordinate system is chosen where $x$ and $y$ denote the circumferential and the axial direction respectively. The mechanical circumference angle is $\varphi=\frac{\pi}{T_{\mathrm{h}}} x$, with $T_{\mathrm{h}}$ being the half of the total spatial period, which is the circumference of the machine. In this plane, the scalar magnetic potential $\phi$ is solved:

$$
\frac{\partial^{2} \phi}{\partial x^{2}}+\frac{\partial^{2} \phi}{\partial y^{2}}=0
$$

The model consists of five regions. Region I and $\mathrm{V}$ represent the area where the PMs are located. Region II and IV are the right and the left air gap, respectively, and Region III represents the stator domain. The stator representation is a solid isotropic cylinder. The thickness $2 \varepsilon$ of the slotless Region III is chosen in such a way that the tangential reluctance of the model equals the reluctance of the real stator with slots. The value of $\varepsilon$ is chosen based on a reluctance model. This homogenized Region III hence accounts for the anisotropy and the presence of slots in the real stator.

The flux density field in the air gaps is solved for a slotless machine with infinite magnetic permeability for the rotor yoke material and adjusted for the slot effect by a permeance function [25].

The magnetization pattern of the PMs is a square wave function with the remanence as peak value for every magnet. By multiplying the healthy remanence with an individual magnetization factor $K_{i}$ for each magnet $i, i=1 \ldots 4 p$ ranging from 0 (demagnetized) to 1 (healthy state), the demagnetization defect is implemented, as shown in Fig. 1(c). $p$ is the number of pole pairs. The reduced remanence of the permanent magnets is considered to be uniformly distributed in the magnet volume. Although partial demagnetization usually occurs non-uniformly, in the scope of condition monitoring, it is more important to evaluate the total flux reduction than to predict exactly which parts of the magnets are demagnetised. The magnetization waves can be described as a Fourier series:

$$
\left\{\begin{array}{l}
M_{1}(x, t)=\sum_{n=-\infty}^{+\infty} M_{1, n} e^{j\left(\frac{n \pi x}{T_{\mathrm{h}}}-\omega n t\right)} \\
M_{2}(x, t)=\sum_{n=-\infty}^{+\infty} M_{2, n} e^{j\left(\frac{n \pi x}{T_{\mathrm{h}}}-\omega n t\right)}
\end{array}\right.
$$

with $\omega$ the pulsation, $M_{1,-n}=M_{1, n}^{*}$ and $M_{2,-n}=M_{2, n}^{*}$. Here, $M_{1}$ and $M_{2}$ represent the magnetization of the rotor in region I and $\mathrm{V}$ respectively. For the magnetic field of the PMs, the time harmonics are the same as the space harmonics, thus the harmonic number for both space and time is $n$. The scalar potentials in the five regions are:

$$
\left\{\begin{aligned}
I: \phi_{1} & =\sum_{n=-\infty}^{+\infty}\left(C_{1} e^{\frac{n \pi y}{T_{\mathrm{h}}}}+C_{2} e^{-\frac{n \pi y}{T_{\mathrm{h}}}}\right) e^{\frac{j n \pi x}{T_{\mathrm{h}}}} \\
I I: \phi_{2} & =\sum_{n=-\infty}^{+\infty}\left(C_{3} e^{\frac{n \pi y}{T_{\mathrm{h}}}}+C_{4} e^{-\frac{n \pi y}{T_{\mathrm{h}}}}\right) e^{\frac{j n \pi x}{T_{\mathrm{h}}}} \\
I I I: \phi_{3} & =\sum_{n=-\infty}^{+\infty}\left(C_{5} e^{\frac{n \pi y}{T_{\mathrm{h}}}}+C_{6} e^{-\frac{n \pi y}{T_{\mathrm{h}}}}\right) e^{\frac{j n \pi x}{T_{\mathrm{h}}}} \\
I V & : \phi_{4}=\sum_{n=-\infty}^{+\infty}\left(C_{7} e^{\frac{n \pi y}{T_{\mathrm{h}}}}+C_{8} e^{-\frac{n \pi y}{T_{\mathrm{h}}}}\right) e^{\frac{j n \pi x}{T_{\mathrm{h}}}} \\
V & : \phi_{5}=\sum_{n=-\infty}^{+\infty}\left(C_{9} e^{\frac{n \pi y}{T_{\mathrm{h}}}}+C_{10} e^{-\frac{n \pi y}{T_{\mathrm{h}}}}\right) e^{\frac{j n \pi x}{T_{\mathrm{h}}}} .
\end{aligned}\right.
$$


The magnetic field can be written as:

$$
H_{x}=-\partial \phi / \partial x, \quad H_{y}=-\partial \phi / \partial y .
$$

The boundary conditions for the problem are:

$$
\begin{aligned}
& \left\{\begin{array}{l}
\left.H_{x \mathrm{I}}(x, y, t)\right|_{[y=L]}=0 \\
\left.H_{x \mathrm{~V}}(x, y, t)\right|_{[y=-L]}=0
\end{array}\right. \\
& \left\{\begin{array}{l}
\left.B_{y \mathrm{I}}(x, y, t)\right|_{[y=\delta]}=\left.B_{y I I}(x, y, t)\right|_{[y=\delta]} \\
\left.H_{x \mathrm{I}}(x, y, t)\right|_{[y=\delta]}=\left.H_{x I I}(x, y, t)\right|_{[y=\delta]}
\end{array}\right. \\
& \left\{\begin{array}{l}
\left.B_{y \mathrm{II}}(x, y, t)\right|_{[y=\varepsilon]}=\left.B_{y I I I}(x, y, t)\right|_{[y=\varepsilon]} \\
\left.H_{x \mathrm{II}}(x, y, t)\right|_{[y=\varepsilon]}=\left.H_{x I I I}(x, y, t)\right|_{[y=\varepsilon]}
\end{array}\right. \\
& \left\{\begin{array}{l}
\left.B_{y \mathrm{III}}(x, y, t)\right|_{[y=-\varepsilon]}=\left.B_{y I V}(x, y, t)\right|_{[y=-\varepsilon]} \\
\left.H_{x \mathrm{III}}(x, y, t)\right|_{[y=-\varepsilon]}=\left.H_{x I V}(x, y, t)\right|_{[y=-\varepsilon]}
\end{array}\right. \\
& \left\{\begin{array}{l}
\left.B_{y \mathrm{IV}}(x, y, t)\right|_{[y=-\delta]}=\left.B_{y V}(x, y, t)\right|_{[y=-\delta]} \\
\left.H_{x \mathrm{IV}}(x, y, t)\right|_{[y=-\delta]}=\left.H_{x V}(x, y, t)\right|_{[y=-\delta]}
\end{array}\right.
\end{aligned}
$$

By solving this system, i.e. (1) - (5), the constants $C_{1}$ to $C_{10}$ can be found. From this, the flux densities in the air gap regions, i.e. regions II and V, are derived:

$$
\begin{aligned}
& B_{y \mathrm{II}}(x, y, t)=\sum_{n=-\infty}^{+\infty}-\frac{\mu_{0} n \pi}{T_{\mathrm{h}}}\left(C_{3} e^{\frac{n \pi y}{T_{\mathrm{h}}}}-C_{4} e^{-\frac{n \pi y}{T_{\mathrm{h}}}}\right) e^{j\left(\frac{n \pi x}{T_{\mathrm{h}}}-n \omega t\right)} \\
& B_{x \mathrm{II}}(x, y, t)=\sum_{n=-\infty}^{+\infty}-\frac{\mu_{0} j n \pi}{T_{\mathrm{h}}}\left(C_{3} e^{\frac{n \pi y}{T_{\mathrm{h}}}}+C_{4} e^{-\frac{n \pi y}{T_{\mathrm{h}}}}\right) e^{j\left(\frac{n \pi x}{T_{\mathrm{h}}}-n \omega t\right)} \\
& B_{y \mathrm{IV}}(x, y, t)=\sum_{n=-\infty}^{+\infty}-\frac{\mu_{0} n \pi}{T_{\mathrm{h}}}\left(C_{7} e^{\frac{n \pi y}{T_{\mathrm{h}}}}-C_{8} e^{-\frac{n \pi y}{T_{\mathrm{h}}}}\right) e^{j\left(\frac{n \pi x}{T_{\mathrm{h}}}-n \omega t\right)} \\
& B_{x \mathrm{IV}}(x, y, t)=\sum_{n=-\infty}^{+\infty}-\frac{\mu_{0} j n \pi}{T_{\mathrm{h}}}\left(C_{7} e^{\frac{n \pi y}{T_{\mathrm{h}}}}+C_{8} e^{-\frac{n \pi y}{T_{\mathrm{h}}}}\right) e^{j\left(\frac{n \pi x}{T_{\mathrm{h}}}-n \omega t\right)}
\end{aligned}
$$

The solutions from these equations together with the permeance function result in the air gap fields at no-load. The armature reaction is calculated based on [26]. The injected three phase currents result in the current sheet $K_{\mathrm{A}}(\varphi, t)(7)$. This current sheet is used to solve the flux density $B_{\mathrm{AR}}$ for the armature reaction (8). The slotting effect is again accounted for by the permeance function in [25].

$$
\begin{gathered}
K_{\mathrm{A}}(\varphi, t)=\sum_{n=-\infty}^{+\infty} \sum_{k=-\infty}^{+\infty} K_{\mathrm{A}, k, n} e^{j(k \varphi+n \omega t)} \\
\left\{\begin{array}{l}
B_{\mathrm{AR}, y}(\varphi, y, t)=\sum_{n=-\infty}^{+\infty} \sum_{k=-\infty}^{+\infty} B_{\mathrm{AR}, y, k, n} e^{j(k \varphi+n \omega t)} \\
B_{\mathrm{AR}, \varphi}(\varphi, y, t)=\sum_{n=-\infty}^{+\infty} \sum_{k=-\infty}^{+\infty} B_{\mathrm{AR}, \varphi, k, n} e^{j(k \varphi+n \omega t)}
\end{array}\right. \\
\left\{\begin{array}{l}
B_{\mathrm{AR}, y, k, n}=j \mu_{0} \frac{\cosh \left(\frac{k}{r} y_{1}\right)}{\sinh \left(\frac{k}{r} y_{2}\right)} \cosh \left(\frac{k}{r}\left(y_{2}-y\right)\right) K_{\mathrm{A}, k, n} \\
B_{\mathrm{AR}, \varphi, k, n}=\mu_{0} \frac{\cosh \left(\frac{k}{r} y_{1}\right)}{\sinh \left(\frac{k}{r} y_{2}\right)} \sinh \left(\frac{k}{r}\left(y_{2}-y\right)\right) K_{\mathrm{A}, k, n}
\end{array}\right.
\end{gathered}
$$

Here, $k$ is the space harmonics number and $n$ is the time harmonic number of the current sheet and the armature reaction current flux density field. The PM and armature reaction flux densities are superimposed to find the flux linkage in the stator coils, which leads to the phase back-EMFs. Together with the slot leakage inductances $L_{\text {slot }}$, the end winding inductances $L_{\mathrm{end}}$ and the armature resistances $R_{\mathrm{S}}$, the three phase terminal voltages are obtained:

$$
V_{\mathrm{a}, n}=E_{\mathrm{a}, n}+R_{\mathrm{s}} I_{\mathrm{a}, n}+j n \omega_{\mathrm{m}}\left(L_{\mathrm{end}}+L_{\mathrm{slot}}\right) I_{\mathrm{a}, n}
$$

where the inductances are computed based on [27]. As the scalar potential solution can be computed for each harmonic separately, it is possible to calculate only the frequencies of interest. This will reduce CPU times as shown in section 4.2 .
Table 1 Characteristics and parameters of the 3 phase, 8 pole pair, 15 stator slot AFPMSM.

\begin{tabular}{lr}
\hline \hline Parameter & Value \\
\hline Electrical output power (W) & 4000 \\
Rated speed (rpm) & 2500 \\
Rated torque (Nm) & 15 \\
Pole number & 16 \\
Slot/tooth number & 15 \\
Outer diameter (mm) & 148 \\
Inner diameter (mm) & 100 \\
Axial length (mm) & 88 \\
Magnet type & NdFeB SH40 \\
Magnets remanence (T) & 1.26 \\
Magnet thickness (mm) & 7.0 \\
Magnet relative permeability & 1.05 \\
Rotor back-iron thickness (mm) & 8.0 \\
Air gap width (mm) & 1.0 \\
Slot width (mm) & 12.0 \\
Number of windings & 72 \\
Rated current (A) & 7.0 \\
Stator laminations & Construction steel \\
Rotor back-iron mat. & \\
\hline \hline
\end{tabular}

\subsection{Model validation}

The five-region analytical model is validated for a YASA AFPMSM with 3 phases, 8 pole pairs, and 15 stator slots. The geometric and electromagnetic properties of the considered machine are fully described in Table 1 and [28].

Fig. 2 shows the axial component of the air gap flux density in the right air gap for a healthy machine (a), and for the same machine with a randomly chosen demagnetization pattern in the magnets (b). Also in case of demagnetization defects, the figure shows that the analytical and FEM data agree with each other. The five region model can therefore be considered as sufficiently accurate.

For the same machine, an experimental validation is done as well. Fig. 2 also shows that the terminal voltage waveforms at no-load obtained by the analytical model are confirmed by experiments. The figure shows a comparison for the healthy machine (c) and for the machine with one fully demagnetized PM (d).

Finally, Fig. 3 shows the harmonic content of the terminal voltage, comparing the analytical model, the FEM model and experimental results. Also here, a good correspondence is observed for the machine with one fully demagnetized PM.

\section{Harmonic analysis}

In order to build a fast and accurate model of an AFPMSM in both healthy and faulty conditions, it is essential to determine the set of harmonics that is most sensitive to demagnetization faults. By only evaluating this limited set in the analytical model, the model becomes much faster and is more useful for the inverse problem of condition monitoring. Therefore, in the following paragraphs, first the inverse problem is formulated briefly. Secondly the theoretical framework is presented about expected harmonics in a healthy and faulty machine. Then, via a case study, a practical investigation is done. Finally, a sensitivity analysis of the harmonics in the machine is presented, which is the main goal of this paper.

\subsection{Inverse problem formulation}

As was illustrated in [22], the magnetization coefficients $\mathbf{K}$ of PMs can be identified by solving an IP by interpreting the three phase voltages of the analytical model. Generally speaking, the IP minimizes iteratively the following objective function:

$$
O F=\left\|\mathbf{V}_{\mathrm{abc}, \mathrm{m}}(\mathbf{H})-\mathbf{V}_{\mathrm{abc}, \mathrm{s}}(\mathbf{K}, \mathbf{H})\right\|^{2}
$$

where $\mathbf{V}_{\mathrm{abc}, \mathrm{m}}$ and $\mathbf{V}_{\mathrm{abc}, \mathrm{s}}$ are respectively the measured and the corresponding simulated three phase terminal voltages. The simulated three phase terminal voltages are calculated with the analytical 


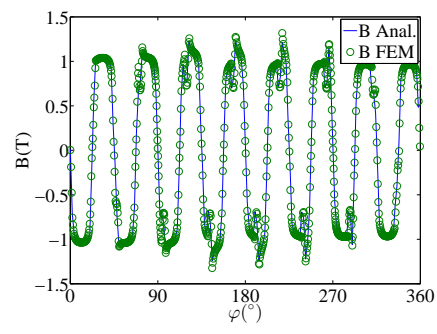

(a)

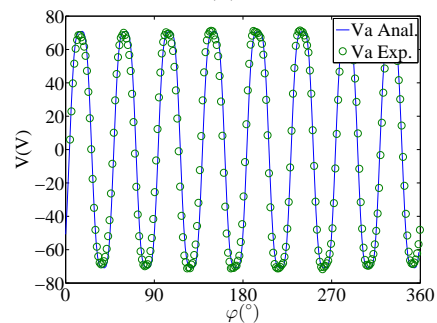

(c)

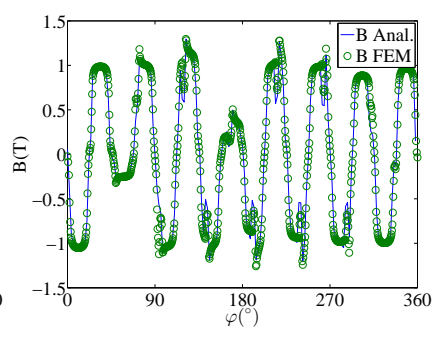

(b)

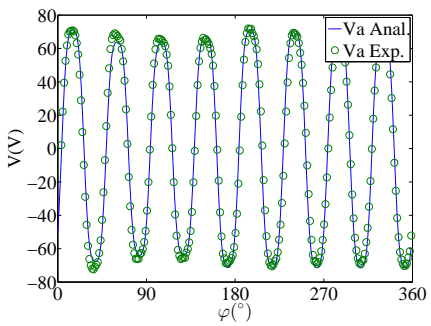

(d)
Fig. 2: Axial component of the air gap flux density in the right air gap for healthy state (a) and for an example demagnetized state (b), calculated by both the analytical and FEM model at full-load. No-load terminal voltage for a speed of $746 \mathrm{rpm}$ in healthy (c) and demagnetized (d) conditions, calculated by the analytical model and compared to experimental data.

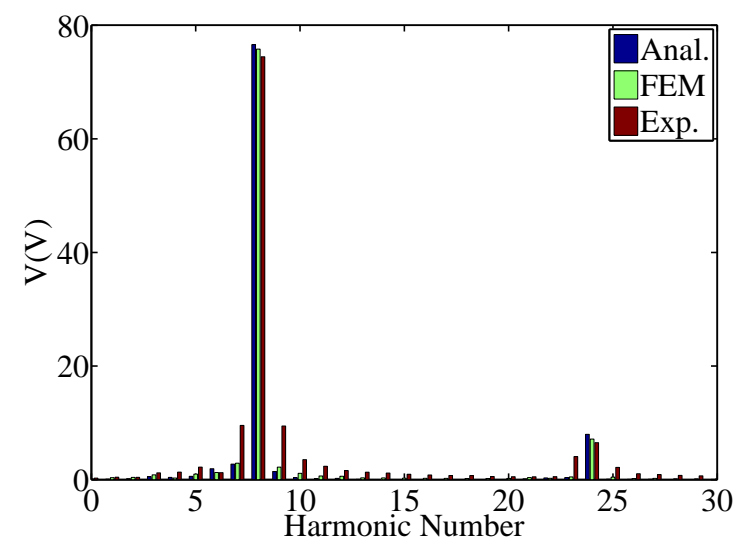

Fig. 3: Harmonic content of the terminal voltage obtained by the analytical model, the FEM and experiments.

model. However, the aim here is not to solve the IP, but to select the optimal set of harmonics $\mathbf{H}$ that speeds up the identification process with keeping the required accuracy.

\subsection{Theoretical framework}

The following analysis is general for any kind of AFPMSMs. We first consider the no-load field of a healthy machine. The space and time harmonics that can occur in such a machine are well known: see e.g. [29]. As mentioned in section 2, because the PM field does not change in time, but only rotates, the occurring space harmonics are the same as the occurring time harmonics and are both denoted by $n$.

In [30], it is stated that in a defected state, the possible harmonics are $n \in \mathbb{Z}_{\neq 0}$.

The underlying principle of which harmonics are sensitive to defects is best shown by considering on the one hand the spectrum of the healthy magnetization pattern $M_{\text {healthy }}(x)$, and on the other hand the spectrum of the "defects waveform" $A_{\mathrm{sq}}(x)$, constructed based on the demagnetization of the magnets. The waveform
Table 2 Winding factors $k_{w n}$ of 3 phase machines with double layer concentrated windings, and (a) 5 pole pairs, 12 stator slots, (b) 8 pole pairs, 15 stator slots.

\begin{tabular}{c||c|c|c|c|c|c|c|c|c}
\hline$n$ & 1 & 2 & 3 & 4 & 5 & 6 & 7 & 8 & 9 \\
\hline (a) $k_{w n}$ & 0.07 & 0 & 0.5 & 0 & 0.93 & 0 & 0.93 & 0 & 0.5 \\
(b) $k_{w n}$ & 0.02 & 0.04 & 0.15 & 0.11 & 0.17 & 0.62 & 0.95 & 0.95 & 0.62 \\
\hline
\end{tabular}

$A_{\text {sq }}(x)$ is equal to one for healthy magnets and smaller than one for partly demagnetized magnets. The product of both waveforms $M_{\text {healthy }}(x)$ and $A_{\mathrm{sq}}(x)$ is the magnetization pattern in case of defects. The above approach becomes more clear via the example in Fig. 4, showing the waveforms in case of a single magnet defect. Fig. 4(a) and (d) show the waveform and the spectrum of the healthy magnetization pattern of the considered YASA machine with 8 pole pairs. The non-zero frequency components have orders given by $n / p=2 a+1, a \in \mathbb{Z}$, but the figure shows only one of these components. Fig. 4(b) and (e) give the waveform and spectrum of the "defects waveform" $A_{\mathrm{sq}}(x)$, which has in this example always value 1 except for the single demagnetized magnet. The resulting demagnetized waveform is the amplitude modulation of the healthy magnetization pattern and the "defects waveform". The waveform and spectrum are given in Fig. 4(c) and (f).

The harmonic spectrum for a sinusoidal magnetization is giving in [30]. This results in a frequency translation over $n=p$ and amplitude scaling with $M_{\text {healthy, } p}$ of the spectrum of $A_{s q}(x)$ as shown in Fig. 4(f).

In general, if $M_{\text {healthy, } n}$ and $A_{\mathrm{sq}, m}$ are the $n$th and $m$ th harmonic of the spectrum of the healthy magnetization pattern and the defect waveform respectively, the defected magnetization pattern can be written as:

$$
\begin{aligned}
M_{\text {demag }}(x) & =M_{\text {healthy }}(x) A_{\mathrm{sq}}(x) \\
& =\sum_{n=-\infty}^{+\infty} M_{\text {healthy }, n} e^{\frac{j n \pi x}{T_{h}}} \sum_{m=-\infty}^{+\infty} A_{\mathrm{sq}, m} e^{\frac{j m \pi x}{T_{h}}} \\
& =\sum_{n=-\infty}^{+\infty} \sum_{m=-\infty}^{+\infty} M_{\text {healthy }, n} A_{\mathrm{sq}, m} e^{\frac{j(n+m) \pi x}{T_{h}}} \\
& =\sum_{n^{\prime}=-\infty}^{+\infty}\left(\sum_{n=-\infty}^{+\infty} M_{\text {healthy }, n} A_{\mathrm{sq}, n^{\prime}-n}\right) e^{\frac{j n^{\prime} \pi x}{T_{h}}} \\
& =\sum_{n^{\prime}=-\infty}^{+\infty} M_{\text {demag, } n^{\prime}} e^{\frac{j n^{\prime} \pi x}{T_{h}}}
\end{aligned}
$$

with $n^{\prime}=n+m$, and $M_{\mathrm{demag}, n^{\prime}}$ as the harmonic components of the demagnetized magnetization pattern. This means that for the considered defect, the multiples of $p$ and it sidebands will be the most sensitive harmonics to this demagnetization defect.

For other demagnetization defects (possibly with more than one demagnetization defect), the amplitude modulation leads to a similar frequency spectrum as the spectrum of the "defect waveform".

Concerning the back-EMF, the orders that occur in the back-EMF spectrum may differ from the harmonic orders in the flux density. Combining the teeth back-EMFs to phase back-EMFs will modify the harmonic spectrum: some harmonics are amplified and others are (partially) cancelled out. An example of this is seen in Fig. 5 where (a) shows one tooth back-EMF and (b) shows one phase back-EMF of a 3 phase, 5 pole pair, 12 stator slot machine.

It is straightforward to determine which harmonics are present in the spectrum of the back-EMF of a given machine, by using the winding factor [27]. In the example machine with 5 pole pairs and 12 stator slots, Table 2 shows the winding factors.

Apart from the no-load field, also the armature reaction field has to be considered. This field can only invoke time harmonics in the stator coils that are in the injected current, therefore only the frequencies in the current need to be calculated for the armature reaction. 


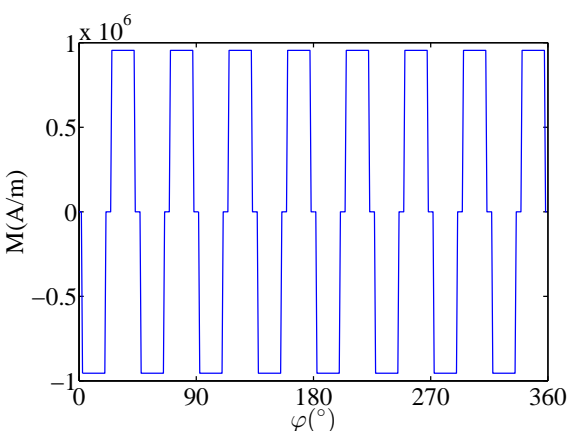

(a)

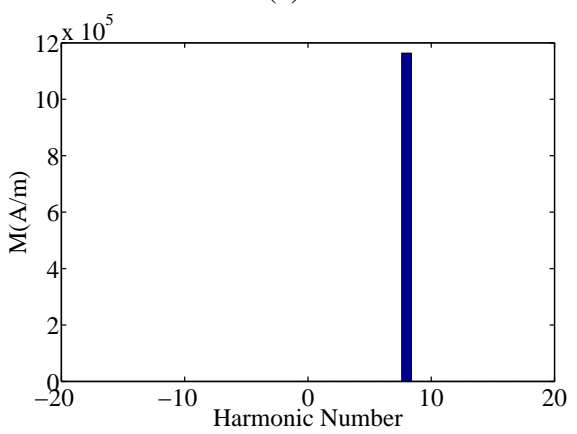

(d)

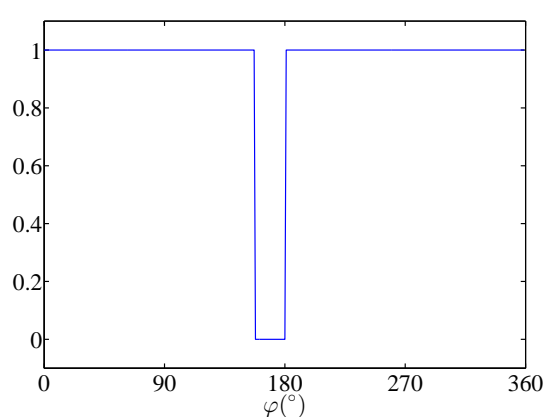

(b)

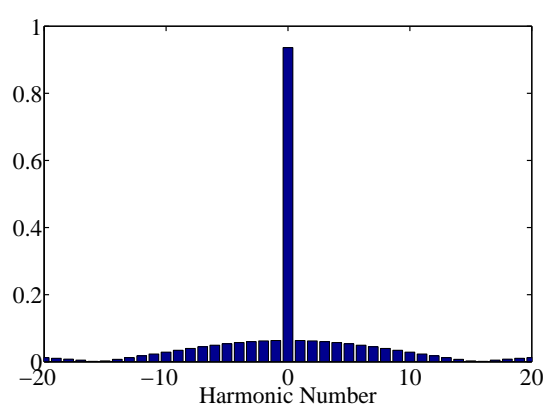

(e)

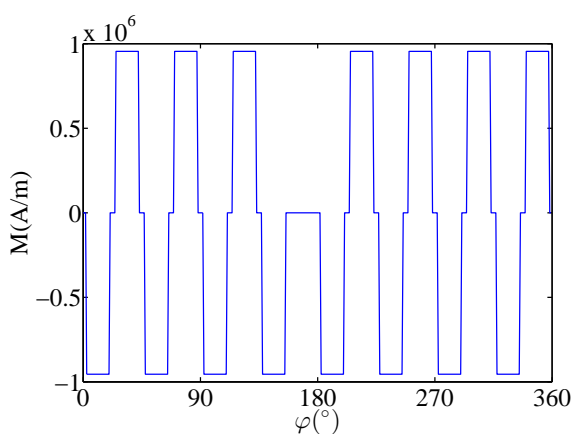

(c)

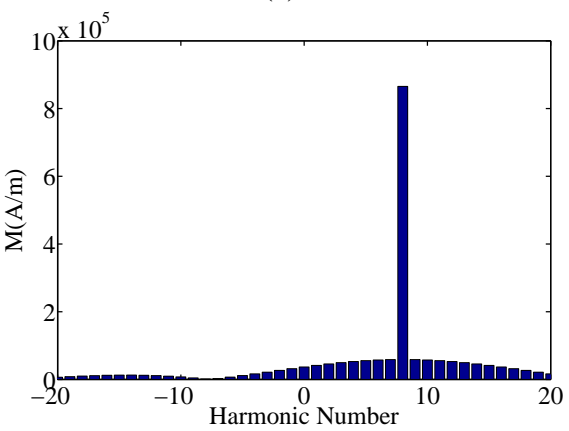

(f)

Fig. 4: (a) The waveform of the healthy magnetization pattern $M_{\text {healthy }}(x)$, (b) the "defects waveform" $A_{s q}(x)$ and (c) the magnetization pattern demagnetized by the "defects waveform" $A_{s q}(x)$. (d),(e) and (f) show the harmonic spectrum of (a),(b) and (c) respectively. For (d) only the $p$ 'th harmonic is considered.

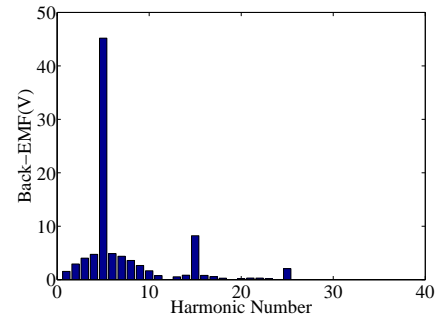

(a)

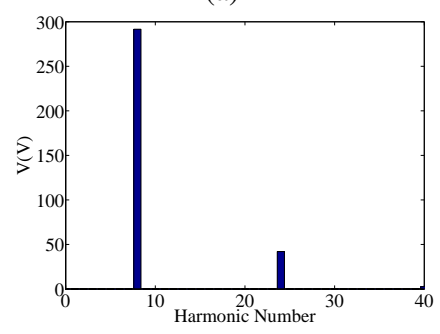

(c)

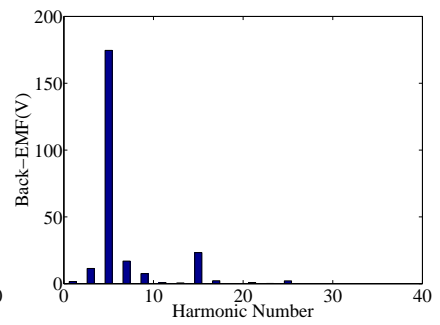

(b)

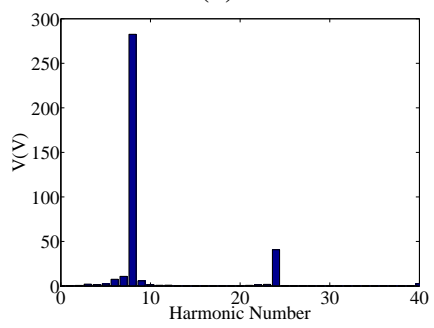

(d)

Fig. 5: The back-EMF of (a) one tooth and (b) one phase. The harmonic spectrum of the terminal voltage at load of the (c) healthy, and (d) faulty state. Both for a 3 phase, 5 pole pair, 12 stator slot machine simulated with the analytical model.

The total set of time harmonics in the terminal voltages is the union of the time harmonics invoked by the permanent magnets and those of the armature reaction.

\subsection{Case study}

By means of example, the harmonic content of the terminal voltages of a specific machine is evaluated. Here, the same 3 phase, 8 pole

pair, 15 stator slot machine is used. A sinusoidal current is injected at the fundamental frequency of the machine and a defect of one completely demagnetized magnet in the right rotor is applied. The fundamental frequency of the machine has the harmonic number 8 .

From [30] the harmonics $8,24,40, \ldots$ are expected in a healthy machine because of the PM field. The defect will add the side bands of these harmonics. The stator current only has the 8'th time harmonic and consequently does not introduce additional harmonics.

Fig. 5 shows the harmonic content of the terminal voltages in healthy (c) and defected (d) state as described. It can be seen that the appearing harmonics satisfy the conditions presented in section 3.2 .

Next section 4 investigates which harmonics are the most sensitive to a demagnetization defect by implementing the CRLB technique.

\section{Sensitivity Analysis}

\subsection{Cramér-Rao lower bound}

The CRLB is a well-known technique to estimate the sensitivity of an output to a certain input parameter. In this paper, the outputs are the terminal voltages $\mathbf{V}_{\mathrm{abc}}$ and the input parameter is the magnetization array $\mathbf{K}$. The likelihood that the given terminal voltages are the result of a certain $\mathbf{K}$ is presented by the likelihood function $\mathcal{L}\left(\mathbf{K} \mid \mathbf{V}_{\mathrm{abc}}\right)$, which is assumed to have a normal distribution.

The Fisher Information Matrix (FIM) is a indicator of how much information the output $\mathbf{V}_{\text {abc }}$ contains about the defect $\mathbf{K}$, in other words: how sensitive $\mathbf{V}_{\text {abc }}$ is to $\mathbf{K}$ :

$$
\mathrm{FIM}=\mathbb{E}\left\{\left[\frac{\partial}{\partial \mathbf{K}} \ln \left(\mathcal{L}\left(\mathbf{K} \mid \mathbf{V}_{\mathrm{abc}}\right)\right)\right]\left[\frac{\partial}{\partial \mathbf{K}} \ln \left(\mathcal{L}\left(\mathbf{K} \mid \mathbf{V}_{\mathrm{abc}}\right)\right)\right]^{T}\right\}
$$

Where $\mathbb{E}\{x\}$ is "the expected value or mean of $x$ ". Following [23][31], this becomes: 
Table 3 The sensitivity and The Cramér-Rao lower bound per time harmonic for a defect consisting of one $30 \%$ demagnetized magnet in the right rotor of a 3 phase, 8 pole pair and 15 stator slot machine. Ranked from most to least sensitive.

\begin{tabular}{c||c|c|c|c|c|c|c}
\hline$n$ & 7 & 8 & 6 & 9 & 5 & 3 & 23 \\
\hline FIM $\left(10^{3}\right)$ & 86.8 & 64.2 & 43.5 & 21.6 & 3.1 & 2.7 & 2.1 \\
\hline$\sigma_{\mathrm{K}}\left(10^{-3}\right)$ & 3.4 & 3.9 & 4.8 & 6.8 & 18.0 & 19.1 & 21.6 \\
\hline
\end{tabular}

$$
\mathrm{FIM}=\left(\frac{\partial \mathbf{V}_{a b c}(\overline{\mathbf{K}}, \mathbf{H})}{\partial \mathbf{K}}\right) S^{-1}\left(\frac{\partial \mathbf{V}_{a b c}(\overline{\mathbf{K}}, \mathbf{H})}{\partial \mathbf{K}}\right)^{\mathrm{T}}
$$

with $\overline{\mathbf{K}}$ and $S$ being the assumed value of the magnetization coefficients and the variance of the likelihood function, respectively. $S$ is thus an indication of the uncertainty of the model.

Based on the inequality of the Cramér-Rao lower bound theory, the lower bound for the uncertainty of the unknown parameters $\sigma_{\mathbf{K}}$ is given by:

$$
\sigma_{\mathbf{K}}^{2} \geq \mid \text { FIM }\left.\right|^{-1}
$$

$\sigma_{\mathbf{K}}$ is basically a measure for the accuracy of the model. For more information about the CRLB technique, see [32]. Because the analytical model in section 2 can be solved for each frequency separately, both (13) and (14) can be found for every harmonic component. Based on this, the harmonics can be sorted from the most to the least sensitive harmonic.

It is worthy mentioning that the CRLB is a qualitative, not a quantitative, technique. This means that only the ratios of the CRLBvalues are indicative. The results obtained from the CRLB give an indication about the accuracy of the IP. Moreover, the calculation of (13) and (14) are not time demanding since they are based on solving a non-iterative forward problem.

\subsection{A low number of well selected harmonics give good accuracy for the IP}

In order to solve the inverse problem, the number of equations from the forward model needs to be greater than or equal to the number of unknowns. The outputs of the forward model are the terminal voltages, which consist of $m$ phases and $n$ harmonics. Every harmonic per phase is a complex number consisting of two parts, i.e. the real and the imaginary part. Each part leads to an equation for the IP. This results in $2 m n$ equations. The IP solver attempts in finding the magnetization factors $\mathbf{K}$ of the magnets, which are thus the unknowns. When all magnets are considered, this leads to $4 p$ magnets. The condition becomes:

$$
\begin{aligned}
2 m n & \geq 4 p \\
n & \geq \frac{4 p}{2 m} \\
n & \geq n_{\min }=\left\lceil\frac{4 p}{2 m}\right\rceil
\end{aligned}
$$

where $n_{\min }$ is the minimum number of time harmonics required to start solving the inverse problem.

When the previous condition is met, the error in finding the defect, which is $|\mathbf{K}-\hat{\mathbf{K}}|$ with $\hat{\mathbf{K}}$ as the actual defect, reduces when using enough sensitive harmonics. The forward model therefore should compute a minimal number $n_{\text {acc }}$ of harmonics for accurate results, for which $n_{\text {acc }} \geq n_{\min }$. The selection of these harmonics is crucial in order to have the highest possible information about the defect. When selecting $n_{\text {acc }}$ time harmonics for the forward model, the selection can be made (a) by taking the first $n_{\text {acc }}$ harmonics: $0 \rightarrow n_{\text {acc }}-1$ or (b) by selecting the $n_{\text {acc }}$ most sensitive harmonics.

The sensitivity of a harmonic to a demagnetization defect can be found by comparing the FIM values. Sensitive harmonics have higher FIM values. It is interesting to investigate if the inverse problem converges faster if the $n_{\text {acc }}$ most sensitive harmonics are chosen,

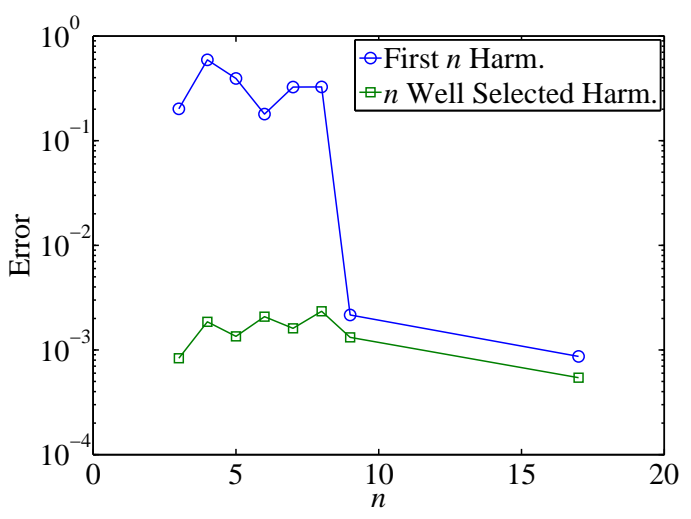

Fig. 6: The accuracy of the IP (using the analytical model) for the first $n$ time harmonics and for $n$ well selected time harmonics for a machine with 8 pole pairs, 15 stator slots and 3 phases.

than if the first $n_{\text {acc }}$ harmonics are chosen. This is first studied for the considered example machine and a given defect. Afterwards, in the following sections, the study is generalized for other defects and other machine topologies.

Table 3 shows the most sensitive harmonics for a defect consisting of one $30 \%$ demagnetized magnet in the right rotor of the considered 3 phase, 8 pole pair and 15 stator slot machine. Because this is a qualitative technique, other defects will result in different FIM values, but a similar sequence of sensitive harmonics.

Fig. 6 compares the accuracy of the IP for both possibilities in this machine. Only the magnets of one rotor are considered in this example ( $2 p$ magnets), so the condition in (15) becomes $n \geq n_{\min }=$ $\left\lceil\frac{2 p}{2 m}\right\rceil$. This means the IP solver can start the calculations only when $n \geq n_{\text {min }}=3$.

The IP was able to solve the problem accurately for $n_{\text {acc }}=9$ if the first $n_{\text {acc }}$ harmonics are chosen and for $n_{\text {acc }}=3$ if the $n_{\text {acc }}$ most sensitive harmonics are chosen. This means the IP needs less harmonics (smaller $n_{\text {acc }}$ ) when using the most sensitive harmonics, which decreases the calculation time, especially when compared to a non-optimized harmonic set (using i.e. high number of harmonics).

\subsection{A set of harmonics can be found to be sensitive for almost all demagnetization defects}

Fig. 7 shows the normalized FIM for 5000 uniformly distributed random defects with resp. 1, 3, 7 and 16 demagnetized magnets for the 8 pole pair, 15 stator slot, 3 phase machine. From these plots, it can be seen that a sequence from high to low sensitivity can be found for every defect specifically.

A general conclusion can be formulated: The multiples of the harmonic $p$ and their sidebands are the most sensitive. The sensitivity tends to decrease with higher multiples of $p$ and with increasing distance from the multiples of $p$.

The magnetization waveform with more demagnetized magnets results in lower sidebands in their spectrum and thus in the FIM spectrum as well. This explains that the sensitivity of the $p$ th harmonic is more distinct for higher numbers of demagnetized magnets.

\subsection{Different topologies: number of poles, slots and phases}

To generalize this conclusion, 16 topologies are compared. 3, 5 and 7 phase machines are considered, with the number of pole pairs ranging from 2 to 9 and the number of stator slots ranging from 10 to 56 . Both fractional-slot as integral-slot topologies are investigated. Fig. 8 shows the normalized FIM for 5000 uniformly distributed random defects of 3 demagnetized magnets for four different topologies.

This figure confirms that certain harmonics are filtered out by combining the tooth back-EMFs to phase back-EMFs by means of the star of slot technique, explained in section 3.2. For example, in Fig. 8(b) all the odd harmonics have a value of zero. 


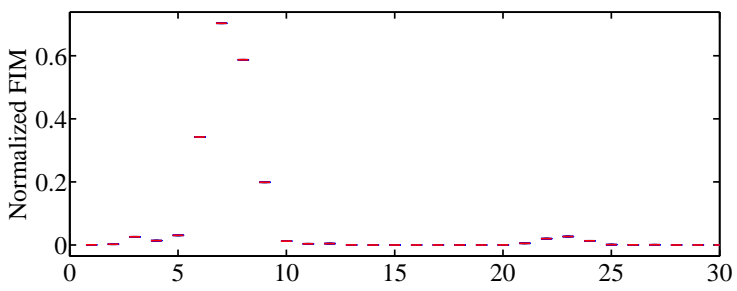

(a) 1 magnet demagnetized

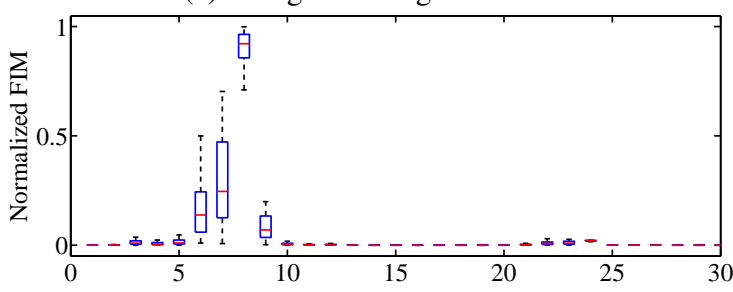

(b) 3 magnets demagnetized

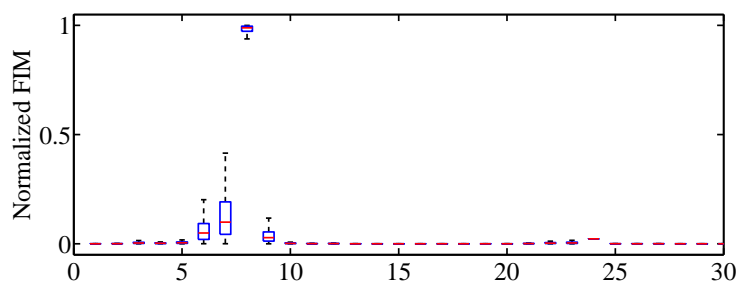

(c) 7 magnets demagnetized

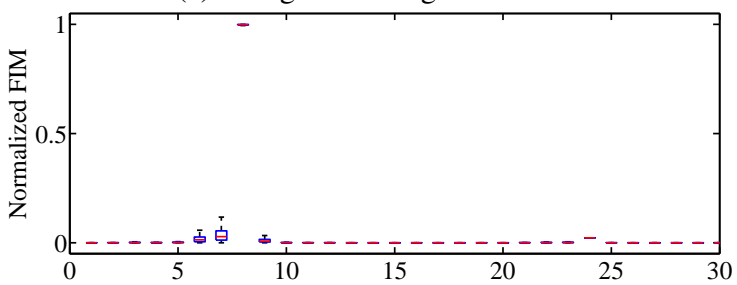

(d) 16 magnets demagnetized

Fig. 7: The normalized FIM of 5000 uniformly distributed random defects of resp. 1, 3, 7 and 16 demagnetized magnets for a machine with 8 pole pairs, 15 stator slots and 3 phases simulated with the analytical model. The harmonic numbers are shown on the $\mathrm{x}$-axis. The box shows the 25 and 75 percentiles while the dashed lines shows the lower and upper adjacent value. The red line is the median. The outliers are not plotted to improve the figures clarity.

Taking this into account, the same conclusion can be drawn for different topologies: the multiples of the harmonic $p$ and their sidebands are the most sensitive, with the sensitivity decreasing with higher multiples of $p$ and with increasing distance from the multiples of $p$.

\subsection{Non-uniform demagnetization defects}

The forward model assumes uniform demagnetization in magnets, but in reality, the demagnetization in magnets may be partial and non-uniform. If a non-uniform demagnetized case is solved with the IP model using the sensitive harmonic array for uniform situations, the decreased flux is detected and the correct region where the demagnetization occurs is found. This was proven by testing the IP for many cases with non-uniform demagnetization. The proposed algorithm is useful for condition monitoring also for real situations with non-uniform demagnetization. However, in the non-uniform cases, part of the demagnetization can be assigned to the neighboring magnets. It should be noted that in more extreme cases where the magnetization waveform is substantially different from the uniform

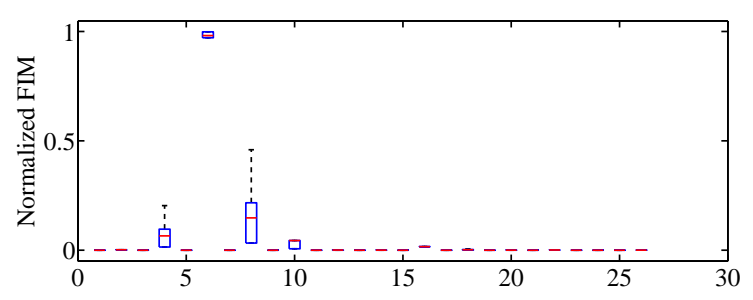

(a) 3 phases, 5 pole pairs and 12 stator slots

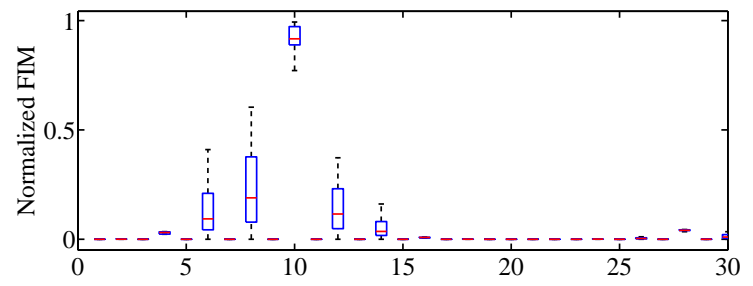

(b) 5 phases, 9 pole pairs and 20 stator slots

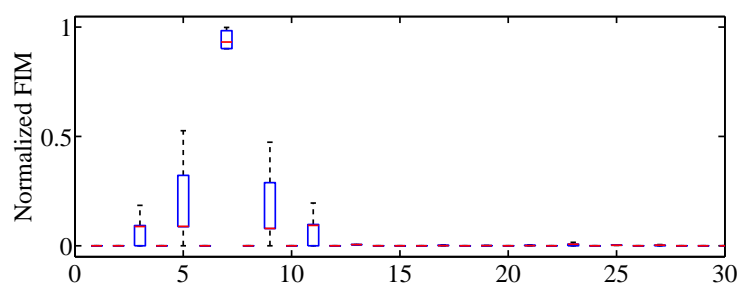

(c) 7 phases, 6 pole pairs and 14 stator slots

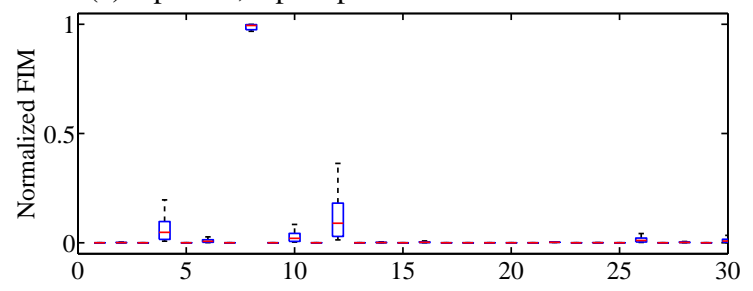

(d) 3 phases, 7 pole pairs and 18 stator slots

Fig. 8: The normalized FIM of 5000 uniformly distributed random defects of 3 demagnetized magnets for four different topologies of double rotor axial flux machines simulated with the analytical model. The harmonic numbers are shown on the $\mathrm{x}$-axis. The box shows the 25 and 75 percentiles while the dashed lines shows the lower and upper adjacent value. The red line is the median. The outliers are not plotted to improve the figures clarity.

situation (especially with many demagnetized magnets that all have totally different magnetization patterns), the harmonic subset will be too different. In these cases, the proposed technique cannot be used.

\section{Conclusion}

The aim of this paper is to determine the optimal set of time harmonics that needs to be simulated for a fast and accurate detection of PM demagnetization faults in the AFPMSM. An analytical model of an AFPMSM is built that allows the calculation of every frequency component independently. The model is validated with FEM and experimental data. The theoretical framework and practical case study conclude that the multiples of the harmonic $p$ and their sidebands are the most sensitive, with the sensitivity decreasing with higher multiples of $p$ and with increasing distance from the multiples of $p$. The sensitivity analysis of the Cramér-Rao lower bound technique confirms this conclusion, even for different topologies. If the first $n$ harmonics are used, given the high sensitivity of harmonic $p$, it seems logic to select the harmonics up to at least order $p$. If the 
most sensitive harmonics are used, the minimum amount of harmonics for the solver to start the calculations is in most cases also enough to find an accurate solution: $n_{\text {acc }}=n_{\min }$.

\section{Acknowledgments}

The authors gratefully acknowledge the financial support of the Special Research Fund "Bijzonder Onderzoeksfonds" of Ghent University.

\section{References}

1 F. G. Capponi, et al., "Recent advances in axial-flux permanent-magnet machine technology," IEEE Trans. Ind. Appl., vol. 48, no. 6, pp. 2190-2205, Nov./Dec. 2012.

2 A. Mahmoudi, et al., "Design and prototyping of an optimised axial-flux permanent-magnet synchronous machine," IET Electr. Power Appl., vol. 7, no. 5, pp. 338-349, 2013

3 T. J. Woolmer, et al., "Analysis of the yokeless and segmented armature machine," International Electric Machines and Drives Conference (IEMDC), 2007, vol. 1, pp. 704-708, 2014.

4 S. M. Mirimani, et al., "Effect of inclined static eccentricity fault in single statorsingle rotor axial flux permanent magnet machines," IEEE Trans. Magn., vol. 48, no. 1, pp. 143-149, Jan. 2012.

5 P. J. Tavner, "Review of condition monitoring of rotating electrical machines," IET Electr. Power Appl., vol. 2, no. 4, pp. 215-247, 2008.

6 R. Fratila, et al., "Nonlinear modeling of magnetization loss in permanent magnets," IEEE Trans. Magn., vol. 48, no. 11, pp. 2957-2960, Nov. 2012.

7 K.-D. Lee, et al., "Local demagnetisation analysis of a permanent magnet motor," IET Electr. Power Appl., vol. 9, no. 3, pp. 280-286, 2015.

8 J. Faiz and H. Nejadi-Koti, "Demagnetization Fault Indexes in Permanent Magnet Synchronous Motors-An Overview," IEEE Trans. Magn., vol. 52, no. 4, Apr. 2016.

9 S. S. Moosavi, et al., "Demagnetization fault diagnosis in permanent magnet synchronous motors: A review of the state-of-the-art," J. Magn. Magn. Mater., vol. 391, pp. 203-212, 2015.

10 T. Goktas, M. Zafarani, and B. Akin, "Discernment of Broken Magnet and Static Eccentricity Faults in Permanent Magnet Synchronous Motors" IEEE Trans. Energy Convers., vol. 31, no. 2, pp. 585-594, 2016.

11 J. Park and J. Hur, "Detection of Inter-Turn and Dynamic Eccentricity Faults Using Stator Current Frequency Pattern in IPM-Type BLDC Motors," IEEE Trans. Ind. Electron., vol. 63, no. 3, pp. 1771-1780, 2016.

12 S. M. Mirimani, A. Vahedi, F. Marignetti, and E. De Santis, "Static Eccentricity Fault Detection in Single-StatorâĂŞSingle-Rotor Axial-Flux Permanent-Magnet Machines," IEEE Trans. Ind. Appl., vol. 48, no. 6, pp. 1838-1845, 2012.

13 M. Zafarani, T. Goktas, and B. Akin, "A Comprehensive Analysis of Magnet Defect Faults in Permanent Magnet Synchronous Motors," in Applied Power Electronics Conference and Exposition (APEC), 2015, pp. 2779-2783.

14 J. C. Urresty, J. R. Riba, and L. Romeral, "A back-emf based method to detect magnet failures in PMSMs," IEEE Trans. Magn., vol. 49, no. 1, pp. 591-598, 2013.

15 Y. Da, X. Shi, and M. Krishnamurthy, "A New Approach to Fault Diagnostics for Permanent Magnet Synchronous Machines Using Electromagnetic Signature Analysis," IEEE Trans. Power Electron., vol. 28, no. 8, pp. 4104-4112, 2013.

16 B. M. Ebrahimi, et al., "Demagnetization Fault Diagnosis in Surface Mounted Permanent Magnet Synchronous Motors," IEEE Trans. Magn., vol. 49, no. 3, 2, pp. 1185âĂȘ1192, 2013.

17 J. F. Bangura, et al., "Diagnostics of eccentricities and bar/end-ring connector breakages in polyphase induction motors through a combination of time-series data mining and time-stepping coupled FE-State-Space techniques," IEEE Trans. Ind. Appl., vol. 39, no. 4, pp. 1005âĂȘ1013, 2003.

18 S. Rajagopalan, et al., "Dynamic eccentricity and demagnetized rotor magnet detection in trapezoidal flux (Brushless DC) motors operating under different load conditions," IEEE Trans. POWER Electron., vol. 22, no. 5, pp. 2061âĂŞ2069, Sep. 2007.

19 H. A. Toliyat et al., Handbook of Electric Motors, 2nd ed. New York, NY, USA: Marcel Dekker, 2004.

20 M. Sasic, et al., "Detecting Turn Shorts in Rotor Windings: A New Test Using Magnetic Flux Monitoring" IEEE Ind. Appl. Mag., vol. 19, no. 2, pp. 63-69, 2013.

21 S. M. Mirimani, A. Vahedi, F. Marignetti, and R. Di Stefano, "An Online Method for Static Eccentricity Fault Detection in Axial Flux Machines," IEEE Trans. Ind. Electron., vol. 62, no. 3, pp. 1931-1942, 2015.

22 J. De Bisschop, et al., "Identification of demagnetization faults in axial flux permanent magnet synchronous machines using an inverse problem coupled with an analytical model," IEEE Trans. Magn., vol. 50, no. 11, Art. No. 8104804, Nov. 2014.

23 A. Abdallh, et al., "Selection of measurement modality for magnetic material characterization of an electromagnetic device using stochastic uncertainty analysis," IEEE Trans. Magn., vol. 47, no. 11, pp. 4564-4573, Nov. 2011.

24 A Hemeida and P. Sergeant "Analytical modeling of surface PMSM using a combined solution of Maxwell's equations and magnetic equivalent circuit (MEC)," IEEE Trans. Magn., vol. 50, no. 12, Art. No. 7027913, Dec. 2014.

25 D. Zarko, et al., "Analytical calculation of magnetic field distribution in the slotted air gap of a surface permanent-magnet motor using complex relative air-gap permeance," IEEE Trans. Magn., vol. 42, no. 7, pp. 1828-1837, 2006.

26 H. Vansompel, et al., "A Multilayer 2-D - 2-D Coupled Model for Eddy Current Calculation in the Rotor of an Axial-Flux PM Machine," IEEE Trans. Energy Convers., vol. 27, no. 3, pp. 784-791, 2012.

27 J. Pyrhönen, et al., Design of rotating electrical machines. New York, NY, USA: Wiley, 2008.
28 H. Vansompel, et al., "Axial-Flux PM Machines With Variable Air Gap," IEEE Trans. Ind. Electron., vol. 61, no. 2, pp. 730-737, 2014

29 B. Hannon, et al., "Harmonic study of a diametrically wound synchronous machine," IEEE Young Researcher Symposium, 2014

30 J.-C. Urresty, et al.,"Detection of Demagnetization Faults in Surface-Mounted Permanent Magnet Synchronous Motors by Means of the Zero-Sequence," IEEE Trans. Energy Convers., vol. 27, no. 1, pp. 42-51, 2012.

31 A. Abdallh, G. Crevecoeur, and L. Dupré, "Optimal needle placement for the accurate magnetic material quantification based on uncertainty analysis in the inverse approach," Meas. Sci. Technol., vol. 21, no. 11, Nov. 2010.

32 G. Goodwin and R. Payne, "Dynamic system identification. Experiment design and data analysis". Mathematics in Science and Engineering, vol. 136. New York, USA: Academic Press, 1977. 